\title{
Antimicrobial susceptibility patterns of uropathogens isolated from pregnant women in KwaZulu-Natal Province: 2011 - 2016
}

\author{
P Bhola, ${ }^{1,2}$ MB ChB, FCPath (SA) Micro, PhD (Med Micro); N R Mvelase, ${ }^{1,2}$ MB ChB, Dip HIV Man (SA), FCPath (SA) Micro, MMed (Micro); \\ Y Balakrishna, ${ }^{3}$ BSc, BSc Hons Statistics, MSc Statistics; K P Mlisana, ${ }^{1,2}$ MB ChB, MMed (Path Micro), PhD (Med Micro); \\ K Swe Swe-Han, ${ }^{1,2}$ MBBS, DTMH, PDIC, FCPath (SA) Micro, MMed (Micro), PhD (Med Micro) \\ ${ }^{1}$ Department of Medical Microbiology, National Health Laboratory Service, Durban, South Africa \\ ${ }^{2}$ School of Laboratory Medicine and Medical Sciences, College of Health Sciences, University of KwaZulu-Natal, Durban, South Africa \\ ${ }^{3}$ Biostatistics Unit, South African Medical Research Council, Durban, South Africa
}

Corresponding author: P Bhola (bholap@ukzn.ac.za)

\begin{abstract}
Background. Urinary tract infection (UTI) is one of the most common infections during pregnancy, which can lead to significant maternal and perinatal morbidity and mortality if left untreated. Challenges when treating UTIs in pregnancy include fetal protection and resistance development of uropathogens. Currently, the Essential Medicines List recommends nitrofurantoin to treat cystitis and ceftriaxone to treat pyelonephritis in pregnant women.

Objectives. To determine common pathogens causing UTI in pregnancy and their antibiotic susceptibility patterns.

Methods. A retrospective analysis was performed of laboratory data for positive urine specimens from obstetric departments of 6 KwaZuluNatal Province hospitals during 2011 - 2016. Identification and susceptibility testing were performed using the VITEK 2 system. Results were interpreted according to the breakpoints of the Clinical and Laboratory Standards Institute, USA.

Results. From 5971 positive urine specimens, the most common isolate was Escherichia coli ( $n=3$ 236; 54.2\%), followed by Klebsiella pneumoniae ( $n=770 ; 12.9 \%)$. Group B streptococcus (GBS) $(n=239 ; 4.0 \%)$ and Enterococcus faecalis $(n=251 ; 4.2 \%)$ were the most common Gram-positive pathogens. E. coli displayed significant resistance to trimethoprim-sulfamethoxazole (65.1\%), cephalothin (38.3\%), cefuroxime (27.3\%), ciprofloxacin (16.9\%) and amoxicillin-clavulanic acid (17.1\%). Resistance to ceftriaxone and nitrofurantoin remained low $-9.1 \%$ and $7.7 \%$, respectively. Among Gram-positive pathogens, GBS displayed 100\% penicillin susceptibility and E. faecalis showed $92.9 \%$ susceptibility to ampicillin.

Conclusions. E. coli is unsurprisingly the most common cause of UTI in pregnancy in KwaZulu-Natal. Susceptibility to ceftriaxone and nitrofurantoin remains good. Among Gram positives, GBS is prevalent and susceptible to penicillin, while E. faecalis is susceptible to ampicillin. As antimicrobial resistance evolves, routine surveillance is necessary to modify recommended empirical antibiotic use.
\end{abstract}

S Afr Med J 2020;110(9):872-876. https://doi.org/10.7196/SAMJ.2020.v110i9.14468

Urinary tract infection (UTI) is one of the most common infections that occurs during pregnancy. If left untreated, it could lead to significant maternal and perinatal morbidity and mortality. ${ }^{[1-3]}$ It may be symptomatic or asymptomatic, which often makes diagnosis difficult. In a large number of patients, UTIs are preceded by asymptomatic bacteriuria (ASB)..$^{[1,4-6]}$ Bacteriuria during pregnancy is associated with a low birthweight and premature delivery if left untreated. ${ }^{[7]}$

The varying prevalence of UTI in pregnancy has been reported worldwide, ranging from $2 \%$ to $10 \% .{ }^{\left[{ }^{[8]}\right.}$ Some studies in the UK have shown that the incidence of ASB in pregnant women ranges from $2 \%$ to $5 \% \cdot{ }^{[9,10]}$ However, the incidence of acute cystitis was more difficult to determine, as most women are treated empirically without culture being performed routinely. ${ }^{[8]}$ Studies conducted in developing countries have shown that UTI often presents during the first antenatal visit and $<1 \%$ develop bacteriuria following a negative screening in early pregnancy. ${ }^{[8]}$ The treatment of ASB in pregnancy decreases the rate of persistent bacteriuria and the subsequent risk of developing pyelonephritis. ${ }^{[1]}$ Based on this, screening for and treating of ASB in high-income countries are considered standard obstetrical care. ${ }^{[1]}$ However, in South Africa (SA) and other resource-limited countries, the cost of standard urine culture is a limiting factor for generalised urine screening. Therefore, other more economical screening methods have been proposed, e.g. urine dipsticks, which is the recommended antenatal screening method in the SA public sector.

During pregnancy, significant physiological changes of the urogenital tract occur, which could increase the risk of pathogenic colonisation. ${ }^{[10]}$ Detrusor tone decreases, bladder volume increases, and a majority of pregnant women develop ureteric dilatation owing to a combination of pressure from an expanding uterus and progestogenic relaxation of ureteric smooth muscle. This ultimately leads to urine stasis and vesico-ureteral reflux, which facilitates bacterial colonisation and ascending infection. ${ }^{[10]}$

Organisms that cause UTI in pregnant and non-pregnant patients are similar. ${ }^{[10]}$ These organisms are usually from normal vaginal, perineal and faecal flora. ${ }^{[8]}$ Common organisms include Escherichia coli, Staphylococcus aureus, Enterococcus faecalis, Proteus mirabilis, Klebsiella pneumoniae and group B streptococcus (GBS), among other less common organisms. ${ }^{[8]}$ Vaginal colonisation with GBS is strongly associated with preterm rupture of membranes, labour and delivery and is a well-established cause of neonatal sepsis. ${ }^{[10]}$ 
Therefore, diagnosing and appropriately treating patients who are infected with these organisms are of paramount importance.

The development of resistance to previously effective antibiotics by common uropathogens has been reported globally in the past few years. ${ }^{[7,11,12]}$ Antibiotic susceptibility patterns vary geographically, as well as in time. ${ }^{[7]}$ Determining the common pathogens associated with UTIs in pregnancy and their antibiotic susceptibility patterns potentially reduces inappropriate antibiotic prescription and therefore development of resistance. ${ }^{[7]}$ Furthermore, detecting the changing susceptibility pattern of uropathogens against commonly used and recommended antibiotics, will be an effective strategy for empirical therapy. ${ }^{[7,13]}$

In SA, the National Department of Health publishes a Standard Treatment Guideline (STG) and Essential Medicines List (EML), which aim to provide clear guidance to healthcare workers regarding the management of all patients at primary care level. ${ }^{[14]}$ Currently, the EML recommends nitrofurantoin to treat cystitis and ceftriaxone for pyelonephritis in pregnant women. Despite these recommendations, there is a paucity of data on the organisms isolated from pregnant women and the antimicrobial susceptibility profiles. This study was therefore conducted to evaluate the common bacterial causes of UTI in pregnancy, as well as the antibiotic susceptibility patterns of the uropathogens.

\section{Methods}

We conducted a retrospective analysis of laboratory reports for all positive urine specimens submitted from the obstetric departments of 6 public sector hospitals in Durban, KwaZulu-Natal Province, SA, during 2011 - 2016. Data were extracted from the local laboratory information system (TrakCare, SA), collated and reviewed. Duplicate results were excluded to reduce over-representation of any particular susceptibility pattern.

Isolates were identified by an automated VITEK 2 system (bioMérieux, France) and susceptibility testing was performed using the same system. Drugs tested for Gramnegative bacteria were ampicillin/amoxicillin, first-generation cephalosporins (e.g. cephalothin), second-generation cephalosporins (e.g. cefuroxime), third-generation cephalosporins (e.g. ceftriaxone), nitrofurantoin, nalidixic acid, amoxicillin-clavulanic acid, ciprofloxacin, gentamicin, amikacin, trimethoprim-sulfamethoxazole and the carbapenems. Antibiotics tested for Grampositive bacteria were penicillin, ampicillin/ amoxicillin and vancomycin. The antibiotic susceptibility testing results were interpreted according to the Clinical and Laboratory Standards Institute (CLSI) breakpoints for the corresponding year. ${ }^{[15]}$

\section{Data analysis}

Simple data analysis based on the laboratory database was performed. Numerator and denominator were calculated as the number of uropathogens isolated and total number of organisms isolated in the urine specimens of pregnant women presenting with UTI, respectively, during the study period. The prevalence rate is a proportion and has been expressed as a percentage. The susceptibility rate was measured in a similar manner and was expressed as a percentage.

\section{Ethical approval}

Ethical approval was granted by the Research Ethics Committee, University of KwaZuluNatal (ref. no. BE085/12).

\section{Results}

Urine specimens with positive microbiological cultures from 5971 pregnant patients were received during the 6-year period. The most common organism isolated was $E$. coli ( $n=3236 ; 54.2 \%$ ), followed by K. pneumoniae $(n=770 ; 12.9 \%)$. Other Gram-negative organisms, including $P$. mirabilis, accounted for only 620 (10.4\%) specimens. Among the Gram-positive organisms, E. faecalis and GBS were the most common organisms isolated ( $n=251 ; 4.2 \%$ and $n=239 ; 4.0 \%$, respectively). Other Gram positives accounted for only $2.4 \%$. Interestingly, the yeasts, Candida albicans and Candida species, were relatively common organisms isolated (6.8\% and 5.2\%, respectively) (Fig. 1).

Table 1 shows the susceptibility of the commonly used antibiotics to treat UTI in pregnancy. E. coli displays a susceptibility of $34.9 \%$ to trimethoprim-sulfamethoxazole. Susceptibility to the first- and secondgeneration cephalosporins is $61.7 \%$ and $72.7 \%$, respectively. Amoxicillin-clavulanic acid and ciprofloxacin have a susceptibility of marginally $>80 \%$. Nitrofurantoin (for treating cystitis in pregnancy) and the thirdgeneration cephalosporins have a susceptibility pattern of $>90 \%$.

Table 2 shows the susceptibility of Grampositive uropathogens to the commonly used antibiotics. E. faecalis displays 93\%

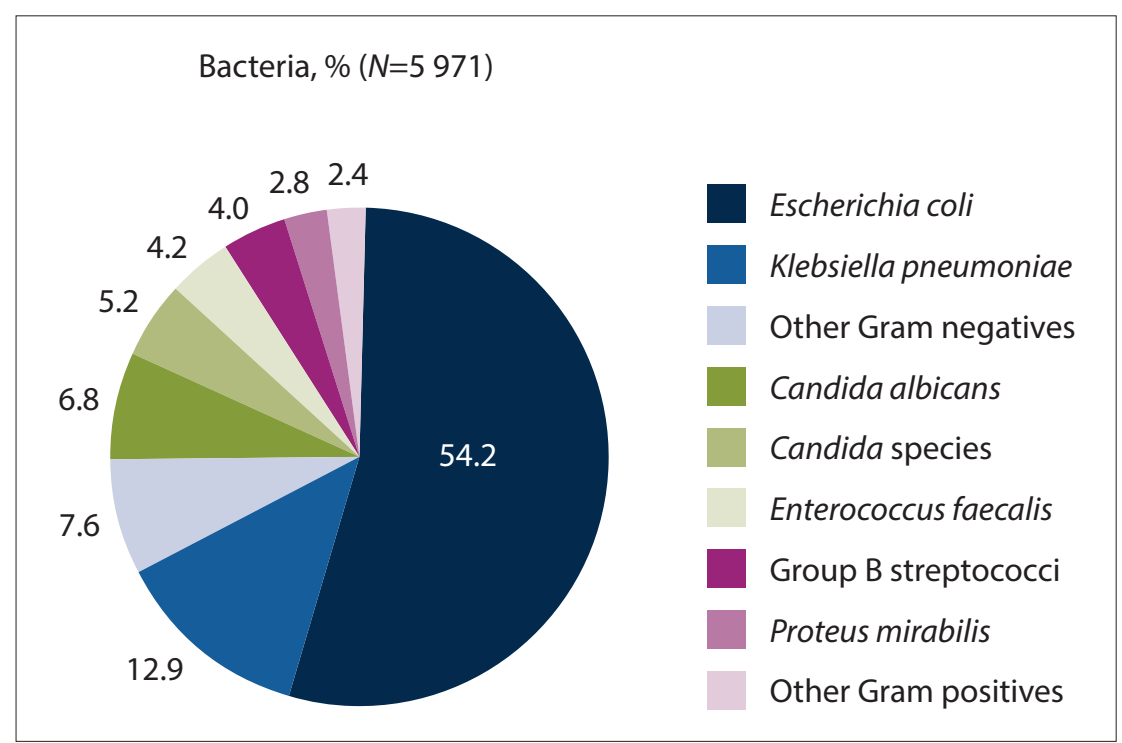

Fig. 1. Frequency of organisms in urine specimens, 2011 - 2016.

Table 1. Susceptibility of Escherichia coli to commonly used antibiotics

\begin{tabular}{|c|c|c|}
\hline \multirow[b]{2}{*}{ Antibiotic } & \multicolumn{2}{|c|}{ Escherichia coli } \\
\hline & Total, $n$ & Susceptible, $n(\%)$ \\
\hline Cephalothin/cephalexin & 1369 & $844(61.7)$ \\
\hline Amoxicillin-clavulanic acid & 2865 & $2376(82.9)$ \\
\hline Cefuroxime & 3253 & $2366(72.7)$ \\
\hline Ceftriaxone/cefotaxime & 2663 & $2419(90.8)$ \\
\hline Trimethoprim-sulfamethoxazole & 2381 & $832(34.9)$ \\
\hline Nitrofurantoin & 2927 & $2701(92.3)$ \\
\hline Ciprofloxacin & 2770 & $2302(83.1)$ \\
\hline
\end{tabular}


susceptibility to ampicillin/amoxicillin (the recommended antibiotic), and GBS is $100 \%$ susceptible to penicillin. The susceptibility to amoxicillin-clavulanic acid is similar, with almost $93 \%$ susceptibility for E. faecalis and $100 \%$ susceptibility for GBS.

Susceptibility of E. coli to amoxicillinclavulanic acid, cefuroxime, ceftriaxone, nitrofurantoin and ciprofloxacin remained consistent over a 6-year period (Fig. 2). A general decline in susceptibility to these antibiotics was observed between 2015 and 2016, with the exception of nitrofurantoin, which remained consistent. Trimethoprimsulfamethoxazole also remained consistent over the 6 years, demonstrating a susceptibility pattern consistently lower than that for the other antibiotics tested.

Line graph values are shown in Table 3.

\section{Discussion}

This study was conducted to determine the common bacterial causes of UTI in pregnancy and to review the susceptibility pattern of drugs that can be used to treat this infection. Data from 6 years revealed E. coli as the most common uropathogen, accounting for $54.2 \%$ of pregnant patients in KwaZulu-Natal. This is in keeping with other studies conducted in both developing and developed countries, where E. coli was found in $35-82 \%$ of cases. ${ }^{[1,16-19]}$ K. pneumoniae was the second most common organism isolated in the urine of obstetric patients, although it was significantly less common than E. coli $(54.2 \%$ v. $12.9 \%)$. Among the Gram-positive organisms, E. faecalis and GBS were the most common organisms isolated $(4.2 \%$ and $4.0 \%$, respectively). In a study by Ulett et al., ${ }^{[20]}$ GBS bacteriuria during pregnancy occurred at a rate of $1-3.5 \%$. $5 \%$ GBS bacteriuria in pregnancy. ${ }^{[21]}$ In this study, GBS accounted for $4.0 \%$ of all isolates in obstetric patients, which is in keeping with the literature. Interestingly, Candida featured prominently ( $12 \%$ collectively), and its significance needs further analysis. With E. coli being the predominant uropathogen detected in this study, which was in keeping with the literature, the susceptibility pattern of antimicrobials was analysed against this organism.

A general decline in susceptibility to the majority of relevant antimicrobials tested was noted in the last 2 years of the study period. This is of increasing concern owing to the already limited options for antimicrobial use in obstetrics and requires close monitoring
Another study showed a prevalence of 0.4 -

and surveillance. Decreasing susceptibility to the cephalosporins and co-amoxicillinclavulanic acid is especially important, as these antibiotics are frequently administered in pregnancy. ${ }^{[14]}$ Ceftriaxone in particular is the antibiotic recommended in the EDL for severe UTI. ${ }^{[14]}$ It is, however, important to note that this antibiotic maintained a susceptibility of $>90 \%$ to $E$. coli during the first 5 years of the study, with a decline to $83.3 \%$ in the last year.

First-generation cephalosporins, e.g. cephalothin/cephalexin, are also very important drugs used in the treatment of UTI. Unfortunately, a disappointing susceptibility of $61.7 \%$ was shown for E. coli during the analysis period. Considering the safety of this antibiotic during pregnancy, as

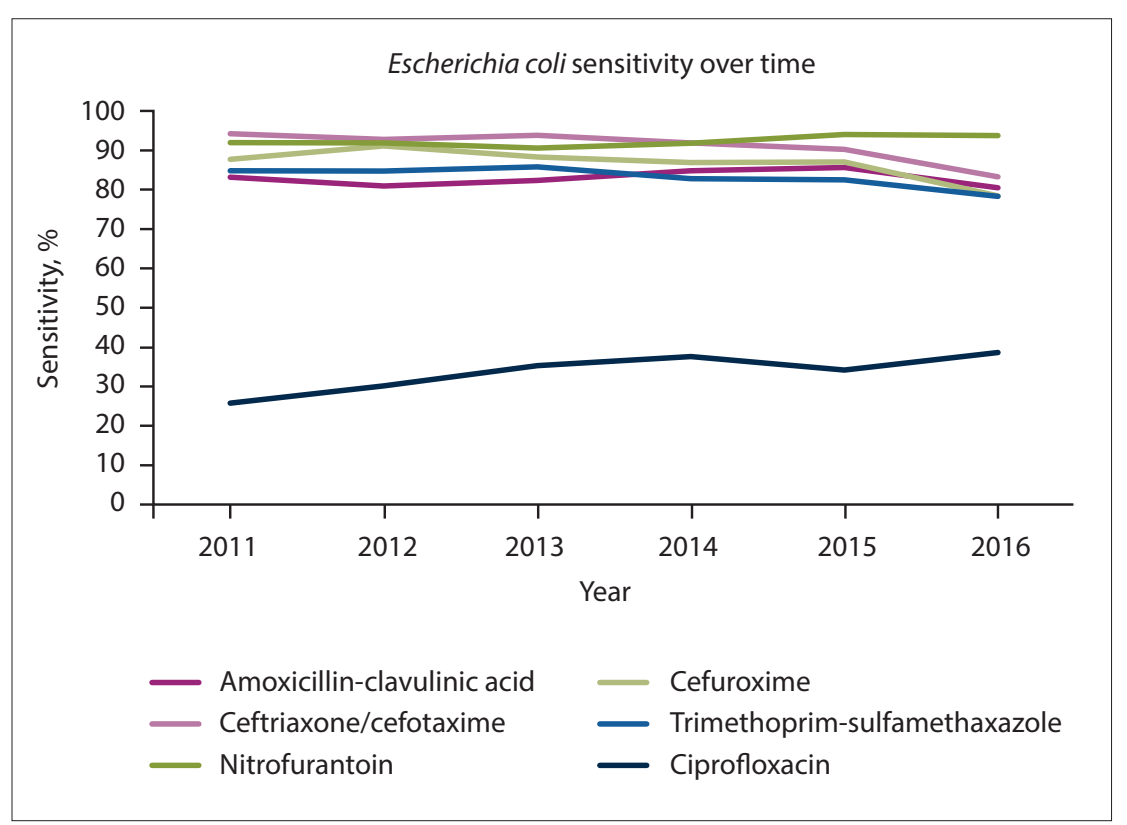

Fig. 2. Susceptibility of Escherichia coli over 6 years.

Table 2. Gram-positive uropathogens

\begin{tabular}{|c|c|c|c|c|}
\hline \multirow[b]{2}{*}{ Antibiotic } & \multicolumn{2}{|c|}{ Enterococcus faecalis } & \multicolumn{2}{|c|}{ Group B streptococci } \\
\hline & Total, $n$ & Susceptible, $n(\%)$ & Total, $n$ & Susceptible, $n(\%)$ \\
\hline Penicillin/ampicillin & 168 & $144(85.7)$ & 221 & $221(100)$ \\
\hline Amoxicillin/ampicillin & 240 & $223(92.9)$ & 148 & $148(100)$ \\
\hline Vancomycin & 205 & $200(97.6)$ & 201 & $196(97.5)$ \\
\hline
\end{tabular}

Table 3. Line graph values for susceptibility of Escherichia coli over 6 years (Fig. 2)

\begin{tabular}{|c|c|c|c|c|c|c|}
\hline \multirow[b]{2}{*}{ Antibiotic } & \multicolumn{6}{|c|}{ Year, \% } \\
\hline & 2011 & 2012 & 2013 & 2014 & 2015 & 2016 \\
\hline Cephalothin/cephalexin & - & - & - & - & 91.7 & - \\
\hline Amoxicillin-clavulanic acid & 83.2 & 80.9 & 82.4 & 84.8 & 85.6 & 80.5 \\
\hline Cefuroxime & 87.7 & 91.1 & 88.3 & 86.9 & 87.1 & 78.4 \\
\hline Ceftriaxone/cefotaxime & 94.2 & 92.8 & 93.8 & 91.8 & 90.2 & 83.3 \\
\hline Trimethoprim-sulfamethoxazole & 25.8 & 30.1 & 35.3 & 37.6 & 34.2 & 38.6 \\
\hline Nitrofurantoin & 92.0 & 91.9 & 90.6 & 91.8 & 94.0 & 93.7 \\
\hline Ciprofloxacin & 84.8 & 84.7 & 85.8 & 82.8 & 82.5 & 78.3 \\
\hline
\end{tabular}


well as its relatively low cost compared with other available antibiotics, it has been a useful alternative to other more expensive antibiotics. This was especially important during the period when nitrofurantoin (recommended for cystitis in pregnancy) had become unavailable owing to cessation of local production. ${ }^{[22]}$ However, this susceptibility pattern may be unreliable, as the testing of nitrofurantoin was not consistent in the participating laboratories, and therefore these results may not be a true reflection of the actual susceptibility to this drug.

Even though E. coli showed an increase in susceptibility to trimethoprim-sulfamethoxazole during the last 2 years of the study, it remains irrelevant because of its low susceptibility pattern of 25 $36.8 \%$ over the study period of 6 years and because it is no longer recommended in SA and many other countries globally for the treatment of UTI. ${ }^{[22-24]}$ A resistance rate of $20 \%$ had previously been recommended as the threshold to avoid treatment with trimethoprimsulfamethoxazole. ${ }^{[23,24]}$ The same study also indicates that this antimicrobial may remain effective at a clinical cure rate of $85 \%$, even when the resistance rate is $30 \% .{ }^{[23]}$ However, insufficient data are available to determine whether the likelihood of failure due to the resistance levels outweighs the benefits of other antimicrobials that are used to treat UTI. ${ }^{[22]}$ Data from our study show a consistently low susceptibility to trimethoprim-sulfamethoxazole, and considering the availability of other potential antibiotics in our setting, this drug has gradually lost its use in the treatment of UTI.

Nitrofurantoin, a urinary antiseptic agent, has maintained a susceptibility pattern of $>90 \%$ (average $92.3 \%$ ) over the 6-year study period. This is encouraging, as the drug still remains the recommendation for cystitis in pregnancy. ${ }^{[14]}$ It has been suggested that the sustained susceptibility of $E$. coli to nitrofurantoin is due to its limited impact on the normal gut flora and thus limited selection of resistant organisms. ${ }^{[22,25]}$ Various studies have demonstrated increased susceptibility of nitrofurantoin to uropathogens in pregnant women with or without UTI. ${ }^{[8,26,27]}$ Consequently, use of this drug as firstline treatment for cystitis has been recommended in these studies. Nitrofurantoin achieves a high urine concentration, but does not penetrate the renal parenchyma very well; therefore, it is not recommended in the treatment of pyelonephritis. ${ }^{[28]}$ Some reports indicate that nitrofurantoin can be associated with a risk of neonatal haemolytic anaemia if the mother has glucose-6-phosphate dehydrogenase (G6PD) deficiency; it is, therefore, advised that this drug should be used with caution. ${ }^{[2,29,30]}$

Decreasing susceptibility to fluoroquinolones (ciprofloxacin) in all of the laboratories is also of concern, as these are firstline drugs recommended internationally ${ }^{[2,23,31]}$ and in the EDL. However, the use of fluoroquinolones is essentially contraindicated during pregnancy owing to reports of fetal cartilage-development disorders. ${ }^{[2,32]}$ However, a systematic review of prospective, controlled studies showed that the use of fluoroquinolones during the first trimester of pregnancy does not appear to be associated with an increased risk of major malformations after birth, stillbirths, preterm births or low birthweight. ${ }^{[2,33]}$ Also, in the maternal care guidelines (National Department of Health), ciprofloxacin is recommended as an alternative agent in cases of penicillin allergy. ${ }^{[34]}$ More data are needed to establish the safety of fluoroquinolones in pregnancy before prescribing or recommending their routine use.

Significantly less Gram-positive organisms than Gram-negative uropathogens were isolated (10\% v. 90\%). E. faecalis and GBS were the most common Gram-positive organisms isolated $(n=251 ; 4.2 \%$ and $n=239 ; 4.0 \%$, respectively). Other Gram positives accounted for only $2.4 \%$. GBS maintains its $100 \%$ susceptibility to penicillin, the drug of choice for this organism, which is in keeping with various other studies in the literature. ${ }^{[35,36]}$ E. faecalis has a susceptibility pattern of almost $90 \%$ to ampicillin/amoxicillin, the recommended antibiotic for this organism. ${ }^{[37]}$ As a result, the data show that these drugs are the recommended drugs of choice in the treatment of UTI caused by the abovementioned organisms.

\section{Study limitations}

This study has various limitations. As it is laboratory based, we could not distinguish between community- and hospital-acquired infections. Furthermore, the request for a urine culture is clinician dependent; therefore, there may be bias in the selection of patients who require urine cultures. There were insufficient data on firstgeneration cephalosporins from all the participating sites, which could significantly affect the recommendations for the treatment of UTI. There was possible bias in the data because of the discordant reporting of antibiotics in the intermediate susceptible range as resistant. Isolates from urine specimens with minimum inhibitory concentration values that fall between susceptible and resistant breakpoints, may be susceptible because elevated levels of the drug can be achieved through concentration in the urine.

Finally, from this analysis, it can be recommended that nitrofurantoin should be used in the treatment of cystitis in pregnancy and ceftriaxone for pyelonephritis. The other recommended antibiotics may also be used, with an awareness of increasing resistance, and the possibility of considering requesting susceptibility testing. As the treatment of UTI is usually empirical, the possibility of treatment failure increases as the levels of resistance increase, and local choices for empirical therapy become restricted. ${ }^{[22]}$ In view of the increasing resistance to commonly used antimicrobials, we may expect an increasing need for culture and susceptibility testing. It would be important to review whether this is the case in future studies. As drug resistance is evolving, routine surveillance is necessary to provide updated information on recommended antibiotic use.

\section{Declaration. None.}

Acknowledgements. The microbiology pathologists at the relevant KwaZulu-Natal laboratories for providing data on their respective hospitals.

Author contributions. PB: conceptualisation and implementation of the research project, data collection, literature review, writing of the first draft, input and editing of all subsequent drafts and writing of final draft; NRM: input on all drafts, editing and analysis; YB: data analysis, tables and figures; KPM: input on implementation of the project and editing the first draft; KSS-H: input and editing final draft.

Funding. None.

Conflicts of interest. None.

1. Kamgang FdPS, Maise HC, Moodley J. Pregnant women admitted with urinary tract infections to a public sector hospital in South Africa: Are there lessons to learn? S Afr J Infect Dis 2016;31(3):79-83. https://doi.org/10.4102\%2Fsajid.v31i3.82

2. Hummers-Pradier E, Kochen MM. Urinary tract infections in adult general practice patients. Br J Gen Pract 2002;52(482):752-761

3. Gilbert NM, O'Brien VP, Hultgren S, et al. Urinary tract infection as a preventable cause of pregnancy complications: Opportunities, challenges, and a global call to action. Glob Adv Health Med 2013;2(5):59-69. https://doi.org/10.7453\%2Fgahmi.2013.061

4. Matuszkiewicz-Rowińska J, Małyszko J, Wieliczko M. Urinary tract infections in pregnancy: Old and new unresolved diagnostic and therapeutic problems. Arch Med Sci 2015;11(1):67-77. https://doi. org/10.5114/aoms.2013.39202

Wing DA, Fassett MJ Getahun D. Acute pyelonephritis in pregnancy: An 18-year retrospective analysis. Am J Obstet Gynecol 2014;210(3):1-6. https://doi.org/10.1016\%2Fj.ajog.2013.10.006

6. Sheffield JS, Cunningham FG. Urinary tract infection in women. Obstet Gynecol 2005;106(5):1085-1092 https://doi.org/10.1097\%2F01.aog.0000185257.52328.a2

7. Sibi G, Kumari P, Kabungulundabungi N. Antibiotic sensitivity pattern from pregnant women with Sibi G, Kumari P, Kabungulundabungi
urinary tract infection in Bangalore, India. Asian Pac J Trop Med 2014;7(Suppl 1):S116-S120. https:// doi.org/10.1016\%2Fs 1995-7645\%2814\%2960216-9 
8. Onoh R, Umeora O, Egwuatu V, et al. Antibiotic sensitivity pattern of uropathogens from pregnant women with urinary tract infection in Abakaliki, Nigeria. Infect Drug Resist 2013;6:225-233. https:// doi.org/10.2147\%2Fidr.s46002

9. National Antibiotic Surveillance. Surveillance forum - private susceptibility data - July - December 2007. S Afr J Epidemiol Infect 2008;23(2):44-48. https://doi.org/10.1080\%2F10158782.2008.11441312

10. McCormick T, Ashe RG, Kearney PM. Urinary tract infection in pregnancy. Obstet Gynaecol 2008;10(3):156-162. https://doi.org/10.1576\%2Ftoag.10.3.156.27418

11. Shigemura K, Tanaka K, Adachi M, et al. Chronological change of antibiotic use and antibiotic resistance in Escherichia coli causing urinary tract infections. J Infect Chemother 2011;17(5):646-651. https://doi.org/10.1007\%2Fs10156-011-0241-2

12. Gupta K, Hooton TM, Stamm WE. Increasing antimicrobial resistance and the management of uncomplicated community-acquired urinary tract infections. Ann Intern Med 2001;135(1):41-50. https://doi.org/10.7326\%2F0003-4819-135-1-200107030-00012

13. Khan S, Rashmi S, Singh P, et al. Pregnancy-associated asymptomatic bacteriuria and drug resistance. J Taibah Univ 2015;10(3):340-345. https://doi.org/10.1016\%2Fj.jtumed.2015.01.011

14. National Department of Health. Standard Treatment Guideline and Essential Medicine List for South Africa. Primary Health Care Level. Pretoria: NDoH, 2018.

15. Clinical and Laboratory Standard Institute. Performance Standards for Antimicrobial Susceptibility Testing. M100-S21-26. Pennsylvania, USA: CLSI, 2017.

16. Farkash $\mathrm{E}$, Weintraub AY, Sergienko R, et al. Acute antepartum pyelonephritis in pregnancy: A critical analysis of risk factors and outcomes. Eur J Obstet Gynaecol Reprod Biol 2012;162(1):24-27. https:// doi.org/10.1016\%2Fj.ejogrb.2012.01.024

17. Oladeinde $\mathrm{BH}$, Omoregie R, Oladeinde $\mathrm{OB}$. Asymptomatic urinary tract infection among pregnant women receiving ante-natal care in a traditional birth home in Benin city, Nigeria. Ethiop J Health Sci 2015;25(1):3. https://doi.org/10.4314\%2Fejhs.v25i1.2

18. MacLean AB. Urinary tract infection in pregnancy. Int J Antimicrob Agents 2001;17(4):273-277. https://doi.org/10.1016\%2Fs0924-8579\%2800\%2900354-x

19. Unlu B, Yildiz Y, Keles I, et al. Urinary tract infection in pregnant population, which empirical antimicrobial agent should be specified in each of the three trimesters? Ginekol Pol 2014;85(5):371-376. https://doi.org/10.17772\%2Fgp\%2F1744

20. Ulett KB, Benjamin Jr WH, Ulett GC, et al. Diversity of group B streptococcus serotypes causing urinary tract infection in adults. J Clin Microbiol 2009;47(7):2055-2060. https://doi. org/10.1128\%2Fjcm.00154-09

21. Allen VM, Yudin MH. Management of group B streptococcal bacteriuria in pregnancy. J Obstet Gynaecol Can 2018;40(2):e181-e186. https://doi.org/10.1016\%2F.jogc. 2017.11.025

22. Bamford C, Bonorchis K, Ryan A, et al. Antimicrobial susceptibility patterns of Escherichia coli strains isolated from urine samples in South Africa from 2007 - 2011. S Afr J Epidemiol Infect 2012;27(2):46-52. https://doi.org/10.1080\%2F10158782.2012.11441483

23. Gupta K, Hooton TM, Naber KG, et al. International clinical practice guidelines for the treatment of acute uncomplicated cystitis and pyelonephritis in women: A 2010 update. Clin Infect Dis 2011;52(5):e103-e120. https://doi.org/10.1093\%2Fcid\%2Fciq257
24. Raz R, Kennes Y, Colodner R, et al. Empiric use of trimethoprim-sulfamethoxazole (TMP-SMX) in the treatment of women with uncomplicated urinary tract infections, in a geographical area with the treatment of women with uncomplicated urinary tract infections, in a geographical area with a
high prevalence of TMP-SMX-resistant uropathogens. Clin Infect Dis 2002;34(9):1165-1169. https:// high prevalence of TMP-SMX-r

25. Mavromanolakis E, Maraki S, Samonis G, et al. Effect of norfloxacin, trimethoprim-sulfamethoxazole and nitrofurantoin on fecal flora of women with recurrent urinary tract infections. J Chemother 1997;9(3):203-207. https://doi.org/10.1179\%2Fjoc.1997.9.3.203

26. Ezechi OC, Fasubaa OB, Dare FO. Antibiotic sensitivity patterns of microbial isolates from the urin of pregnant women with urinary tract infections. Trop J Obstet Gynaecol 2004;20(2):113-115. https:/ doi.org/10.4314\%2Ftjog.v20i2.14413

27. Assefa A, Asrat D, Woldeamanuel Y, et al. Bacterial profile and drug susceptibility pattern of urinary tract infection in pregnant women at Tikur Anbessa Specialized Hospital Addis Ababa, Ethiopia. Ethiop Med J 2008;46(3):227-235.

28. Clark CJ, Kennedy WA, Shortliffe LD. Urinary tract infection in children: When to worry. Urol Clin N Am 2010;37(2):229-241. https://doi.org/10.1016\%2Fj.ucl.2010.03.009

29. Dwyer PL, O'Reilly M. Recurrent urinary tract infection in the female. Curr Opin Obstet Gynecol 2002;14(5):537-543. https://doi.org/10.1097\%2F00001703-200210000-00016

30. Berard A, Santos F, Ferreira E, et al. Urinary tract infections during pregnancy. In: Tenke P, ed. Urinary Tract Infections, 2011:113-134. https://www.intechopen.com/books/urinary-tract-infections/urinarytract-infections-during-pregnancy (accessed 4 August 2020).

31. Hooton TM, Scholes D, Gupta K, et al. Amoxicillin-clavulanate vs ciprofloxacin for the treatment of uncomplicated cystitis in women: A randomized trial. JAMA 2005:293(8):949-955, https://doi. org/10.1001\%2Fjama.293.8.949

32. Smaill FM, Vazquez JC. Antibiotics for asymptomatic bacteriuria in pregnancy. Cochrane Database Syst Rev 2015;(8):CD000490. https://doi.org/10.1002/14651858.cd000490.pub3

33. Bar-Oz B, Moretti ME, Boskovic R, et al. The safety of quinolones - a meta-analysis of pregnancy outcomes. Eur J Obstet Gynaecol 2009;143(2):75-78. https://doi.org/10.1016\%2Fj.ejogrb.2008.12.007

34. National Department of Health. Guidelines for Maternity Care in South Africa. A Manual for Clinics, Community Health Centres and District Hospitals. 4th ed. Pretoria: NDoH, 2015:172.

35. Kasahara K, Baltus AJ, Lee SH, Edelstein MA, Edelstein PH. Prevalence of non-penicillin-susceptible group B streptococcus in Philadelphia and specificity of penicillin resistance screening methods. J Cli Microbiol 2010;48(4):1468-1469. https://doi.org/10.1128\%2Fjcm.02296-09

36. Bolukaoto JY, Monyama CM, Chukwu MO, et al. Antibiotic resistance of Streptococcus agalactioe isolated from pregnant women in Garankuwa, South Africa. BMC Res Notes 2015;8:364. https://doi. org $/ 10.1186 \% 2 \mathrm{Fs} 13104-015-1328-0$

37. Abdulla FE, Abdulla EM. Antibiotic options for Enterococcus faecalis infections. Pak J Med Sci 2006:22(3):286-290

Accepted 30 March 2020. 